\title{
Temporal Behaviour of Sources in the ROSAT Extreme-Ultraviolet All-Sky Survey
}

\author{
PAUl McGale, J. P. PYE, C. R. BARBER, AND C. G. PAGE \\ X-Ray Astronomy Group, Department of Physics and Astronomy, \\ University of Leicester, Leicester LE1 7RH
}

\begin{abstract}
From a total catalogue of $4792 \mathrm{RE}$ sources, 31 have been found to be variable. All the variable sources are optically identified, with a breakdown by source type as follows: cataclysmic variables 9, late-type (F-M) stars 18, the high-mass X-ray binary Her X-1, the eclipsing binaries Algol and V471 Tau, and the active galaxy Mkn 478. The most highly variable objects in the EUV band are cataclysmic variables. The survey was sensitive to timescales from $\sim 1.5 \mathrm{~h}$ to $\sim 5 \mathrm{~d}$, and observed variability ranges from flare-like events lasting $<1.5 \mathrm{~h}$ to irregular and periodic flux changes over $\sim 0.5-2 \mathrm{~d}$. With the exception of the cataclysmic variables, and possibly a few late-type dK-dMe stars with large flares, the observed variability levels should not substantially affect EUV luminosity functions
\end{abstract}

\section{Introduction}

The Wide Field Camera (WFC) on the ROSAT satellite performed the first all-sky survey at extreme-ultraviolet (EUV) wavelengths over a continuous six-month period starting in 1990 July. The survey was conducted in two wavebands, S1 and S2, covering the ranges $60-140 \AA$ and $110-200 \AA$ respectively. Detailed descriptions of the instrument, survey, and analysis procedures can be found in Sims et al. (1990), Pounds et al. (1993) and Pye et al. (1995).

From an initial analysis of the data a bright source catalogue (BSC), containing 383 sources, was published by Pounds et al. (1993). Recently, an updated and expanded version, the '2RE' Catalogue, has been produced (Pye et al. 1995), taking advantage of better knowledge of the survey data base and improved analysis procedures. The $2 \mathrm{RE}$ Catalogue contains 479 EUV sources. Active, late-type (F-M) stars (LTSs) and hot, white-dwarf stars (WDs) account for most of the optical identifications, 251 and 140 respectively. Other identifications are with cataclysmic variables $(\mathrm{CVs})$, active galactic nuclei (AGNs), B-type stars, X-ray binaries (XRBs) and supernova remnants (SNRs) contributing $18,8,10,3$, and 2 counterparts each. A total of 34 sources has, so far, remained unclassified.

During the data processing associated with the 2RE Catalogue, light-curves were produced for all sources. The information from these light-curves has been summarised in the Catalogue in the form of a "time-variability flag." Of the $312 \mathrm{RE}$ sources reported as variable, all have probable optical identifications, with a breakdown as follows: LTSs 20, CVs 9, high-mass X-ray binaries (HMXB) 1 (Her X-1), AGNs 1 (Mkn 478). Hence, 50 percent of the CVs and $\sim 10$ percent of the LTSs in 2RE are classed as variable. 


\section{Variability Analysis}

Each $2 R E$ light-curve was ' $\chi^{2}$-tested' against the hypothesis of a constant source at the measured mean count rate

$$
\chi^{2}=\sum_{i=1}^{n}\left(C_{i}-\bar{C}\right)^{2} / \sigma_{i}^{2} .
$$

Here, $C_{i}$ is the value of the $i$ th time bin $\left(C_{i} \geq 0\right)$, in counts per second, and $\sigma_{i}^{2}$ its variance. The mean of the $C_{i}$ 's is denoted by $\bar{C}$. This yielded a probability $\mathrm{P}\left(>\chi^{2}\right)$.

A source was only flagged as variable if $\mathrm{P}<0.001$ in one or both wavebands. Also, to reduce further potential spurious variability due to small errors in correcting the raw counts to count rates, the $\chi^{2}$ per degree of freedom $\left(\chi_{\nu}^{2}\right)$ was required to be $>2.0$. Thus 31 2RE sources have been identified as variable by Pye et al. (1995). Simulations show that we should expect no more than $\sim 1$ source in the $2 \mathrm{RE}$ Catalogue to be falsely flagged as 'variable'.

For those 2RE sources which passed the variability criteria, two other statistics were computed: (i) the ratio of maximum to mean count rate, as a measure of flare strength; (ii) a 'normalised', rms variability ( $\epsilon_{\text {rms }}$ e.g., Pallavicini et al. 1990 ), to measure the overall variability level.

$\chi_{\nu}^{2}$ is plotted in Fig. 1. Fig. 2 summarises the EUV variability as a function of source class. For a full discussion on the methods of light-curve production and the test statistics applied see McGale et al. (1995).

\section{Discussion}

As can be seen from Fig. 1, the ability to detect variability in the 2RE sources is clearly flux limited, though this manifests itself in several different ways. For example, a source that is very weak, even undetectable, most of the time, may suddenly flare briefly, for say 1 or 2 ROSAT orbital scans, e.g., 2RE J2047-363 (= HD $197890=$ SAO 212437, Matthews et al. 1994). On the other hand, less dramatic variability can be seen in sources with higher 'quiescent' flux levels, e.g. 2RE J0308+405 (= Algol). In terms of timescales, the survey is sensitive to variability in the range $\sim 1.5 \mathrm{~h}$ to $\sim 5 \mathrm{~d}$ (with some drop in detectability for timescales near $1 \mathrm{~d}$ - the nominal interchange frequency of the EUV filters.) In principle, the WFC is also sensitive to timescales from $\sim 40 \mathrm{~ms}$ (set by the on-board storage of the individual detected events) to $\sim 80 \mathrm{~s}$ (corresponding to one scan of a source through the centre of the detector). However in practice, the source needs to be either very strong or periodic to utilise this information, see e.g. the analysis of Her X-1 (= 2RE J1657+352) by Rochester et al. (1994). Owens et al. (1993) have searched the WFC all-sky survey data for rapid transients ( $<50$ s duration) excluding sky locations near known BSC sources; there were no detections.

\subsection{Cataclysmic Variables}

A striking feature of the results (Figs. 1 and 2) is how clearly the CVs stand out from the other source classes, both in the fraction of sources that are seen to be variable and in the level of their variability. Much follow-up work has already been undertaken on the CVs newly-discovered in the WFC all-sky survey. Watson (1993), has summarised the properties of the new EUV-selected sample. 13 out of the total of $182 \mathrm{RE} \mathrm{CVs}$ are AM Her-type systems (ie. 'polars'); 8 out of 9 of the variable $2 R E$ CVs are AM Her systems. The other variable CV is the dwarf nova SS Cyg, one of two such systems in the 2RE Catalogue. 
S1 Waveband

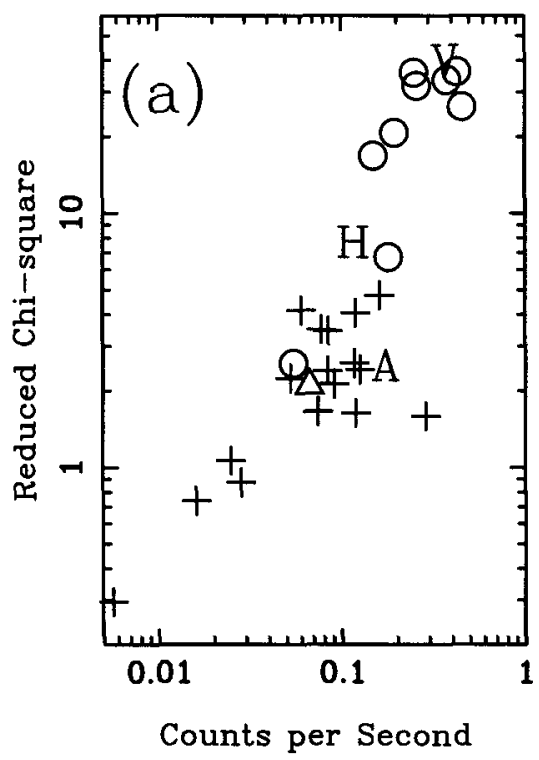

S2 Waveband

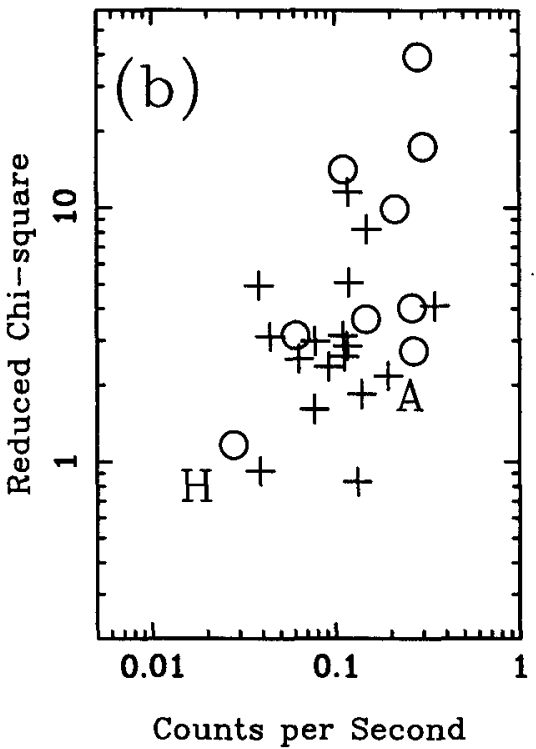

FIGURE 1. Light-curve variability test: $\chi^{2}$ per degree of freedom $\left(\chi_{\nu}^{2}\right)$ versus source mean count rate $\bar{C}$ for reported variable sources only. The symbols $+, O, \Delta$ represent, in turn, $F-M$ stars, CVs and AGNs. Her X-1 is denoted by $\mathrm{H}$, Algol by $\mathrm{A}$ and V471 Tau by V. For the S2 waveband V471 Tau is ont of scale bounds, at $(1.16,288.0)$. (a) S1 band, (b) S2 band.

\subsection{Late-Type Stars}

More than half of the 2RE Catalogue is made up from coronally active stars of spectral types F-M. Of the 18 (or 19 if we include Algol) classified here as EUV-variable, most are also known to be highly variable at optical and/or $\mathrm{X}$-ray wavelengths. There are examples of dMe flare stars, single rapidly rotating dK stars, and RS CVn binary systems. Although it is difficult to make definitive statements due to the limited numbers of objects, the fraction of F-type EUV-variable stars (0/44) appears to be rather lower than for G-M stars (see Fig. 2). Fleming et al. (1995) have found a similar result in the longterm $(\sim 10$ years) behaviour of an X-ray-selected (Einstein Extended Medium Sensitivity Survey) stellar sample. All the G-type EUV-variable stars (9) are either known (7), or possible (2, 2RE J0458+002 = BD+00 908, Strassmeier et al. (1988); 2RE J0106-225 = SAO 166806†) RS CVn systems. Thus 40 percent of the 2 RE G-type RS CVn systems are found to vary. RS CVn systems were also found to be the major identified class of object in the Ariel V sky survey of fast-transient X-ray sources (Pye \& McHardy 1983).

† We suggest 2 RE J0106-225 = SAO 166806 as a possible RS CVn system, and certainly as a very active star, on the following basis. From the G5 V spectral type we estimate a distance of $\approx 33 \mathrm{pc}$, and hence, from the mean S1-band count rate of 0.078 count $\mathrm{s}^{-1}$, an EUV luminosity $L_{\text {EUV }}\left(\mathrm{erg} \mathrm{s}^{-1}\right.$ ) of $\log L_{\mathrm{EUV}} \sim 29.8$ (see Hodgkin \& Pye 1994, Fig. 2a). Such a high value strongly suggests that SAO 166806 is an active spectroscopic binary (see Hodgkin \& Pye 1994, Figs. 5, 6 and 8 ). 
Variable 2RE Sources by Class

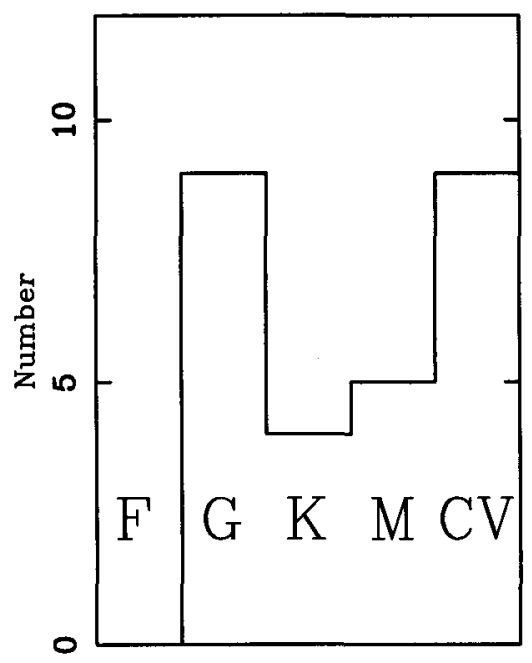

Class
Variable 2RE Sources by Class

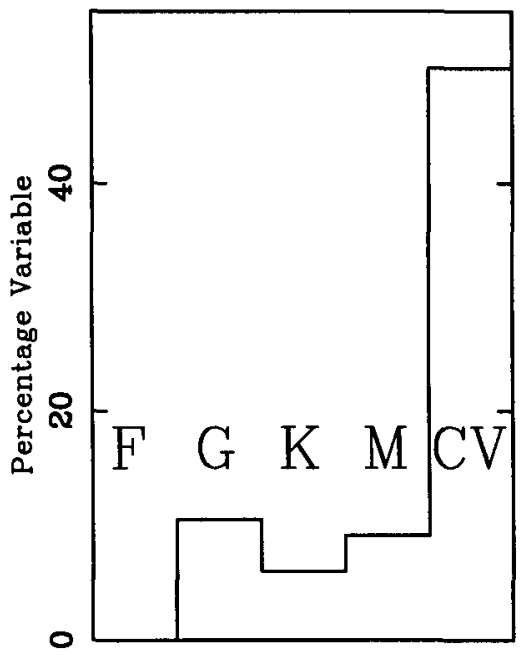

Class

FIGURE 2. Variable 2RE sources by identification class.

Similar variability levels in LTSs have been reported from soft $\mathrm{X}$-ray pointed observations from Einstein and EXOSAT (see e.g. review by Pallavicini 1993, and references therein).

\subsection{Other Source Types}

The eclipsing binary systems V471 Tau (2RE J0350+171, DA + K2 V, detached) and Algol (2RE J0308+405, B8 V + K2 IV, semi-detached) clearly show periodic behaviour in their EUV light-curves, with eclipses seen in both systems (V471 Tau: Barstow et al. 1992, Algol: M. Barstow, private communication). The WFC survey observations of the HMXB Her X-1 have been reported by Rochester et al. (1994). Gondhalekar et al. (1992) have presented WFC survey observations of AGNs, including Mkn 478.

\subsection{Long-Term Variability}

Two years after the WFC survey, the EUV sky was re-examined by NASA's Extreme Ultraviolet Explorer (EUVE, Bowyer et al. 1994). A comparison of the two surveys has been performed by Barber et al. (1995), who find high levels of variability (factors $>10$ ) for CVs, and more modest variability (factors $\sim 2$ ) in the LTSs, during the two years between the surveys. Thus, albeit from this rather limited evidence, the 'short-term' and 'long-term' EUV variability appear similar, though the fraction of LTSs exhibiting variability from the EUVE/WFC comparison may be rather higher, at $\sim 35$ percent, than the $\sim 10$ percent reported here for the shorter timescales. However, this conclusion is tentative given the added uncertainty of cross-calibration between the two surveys. 
The UK ROSAT project is funded by the Particle Physics and Astronomy Research Council.

\section{REFERENCES}

Barber, C. R., Warwick, R. S., McGale, P. A., Pye, J. P., \& Bertram, D. 1995, MNRaS, 273,93

Barstow, M. A., Schmitt, J. H. M. M., Clemens, J. C., Pye, J. P., Denby, M., Harris, A. W., \& PANKIEWICZ, G. S. 1992, MNRAS, 255, 369

Bowyer, S., Lieu, R., Lampton, M., Lewis, J., Wu, X., Drake, J. J., \& Malina, R. F. 1994, ApJS, 93

Fleming, T. A., Molendi, S., Maccacaro, T., \& Wolter, A. 1995, ApJS, in press

Gondhalekar, P. M., Pounds, K. A., Sembay, S., Sokoloski, J., Urry, C. M., Matthews, L., \& QuenBy, J. J. 1992, in Physics of the Active Nuclei, W.J. Duschl \& S.J. Wagner, Springer-Verlag, 52

HODGKIN, S. T., \& PYE, J. P. 1994, MNRAS, 267, 840

Matthews, L., Bromage, G. E., Kellet, B. J., Sidher, S. D., Rochester, G. K., Quenby, J. J., Summer, T. J., O'Donoghue, D., \& Willoughby, G. 1994, MNRAS, 266, 757

McGale, P. A, Pye, J. P., Barber, C. R., \& Page, C. G. 1995, MNRAS, in press

Owens, A., Page, C. G., Sembay, S., \& Schaefer, B. E. 1993, MNRAS, 260, L25

Pallavicini, R. 1993, in Physics of Solar and Stellar Coronae: G.S. Vaiana Memorial Symposium, ed. J.F. Linsky \& S. Serio, Dordrecht: Kluwer, 237

Pallavicini, R., Tagliaferri, G., \& Stella, L. 1990, A\&A, 228, 403

POUNDS, K. A. ET AL. 1993, MNRAS, 260, 77

Pye, J. P. \& MCHARDY, I. M. 1983, MNRAS, 205, 875

PYE, J. P. ET AL. 1995, MNRAS, in press

Rochester, G. K., Barnes, J., Sidher, S., Sumner, T. J., Bewick, A., Corrigan, R., \& QUENBY, J. J. 1994, A\&A, 283, 884

Sims, M. R. ET AL. 1990, Opt. Eng., 29(6), 649

Strassmeier, K. G., Hall, D. S., Zeilik, M., Nelson, E., Eker, Z., \& Fekel, F. C. 1988, A\&AS, Ser., 72, 291

Watson, M. G. 1993, Adv. Space Res., 13, No 12, 12,125 